\title{
Pension Funding Problem with Regime-switching Geometric Brownian Motion Assets and Liabilities
}

\author{
Ping Chen, Hailiang Yang \\ Department of Statistics and Actuarial Science \\ The University of Hong Kong \\ Pokfulam Road, Hong Kong
}

March 12, 2009

\begin{abstract}
This paper extends the pension funding model in Gerber and Shiu (2003) to a regime-switching case. The market mode is modeled by a continuous-time stationary Markov chain. The asset value process and liability value process are modeled by Markov-modulated geometric Brownian motions. We consider a pension funding plan in which the asset value is to be within a band that is proportional to the the liability value. The pension plan sponsor is asked to provide sufficient funds to guarantee the asset value stays above the lower barrier of the band. The amount by which the asset value exceeds the upper barrier will be paid back to the sponsor. By applying differential equation approach, this paper calculates the expected present value of the payments to be made by the sponsor as well as that of the refunds to the sponsor. In addition, we study the effects of different barriers and regime switching on the results using some numerical examples. The optimal dividend problem is studied in our examples as an application of our theory.
\end{abstract}


Keywords: pension funding, regime switching, Markov chain, geometric Brownian motion, system of second order differential equations, optimal dividend problem

\section{Introduction}

Pension funding problem is an important topic for the related companies as well as government. One essential problem is how to match the asset value process and liability value process in a pension plan. Gerber and Shiu (2003) propose to use the strategy which guarantees the pension asset value within a band that is proportional to the pension liability value, where the asset and liability of a company are modeled by correlated geometric Brownian motions with constant drift and instantaneous variance parameters. Since the states of the economy (or "market mode") can be significantly affected by the changes in political policies, the impact of economic news, etc, we may need to model the asset and liability value processes in a way with more flexibility.

In recent years, regime switching models have become popular in finance and related fields. This type of model is motivated by the intension to reflect the state of the financial market. For example, the state of the market can be roughly divided into "bullish" and "bearish" two regimes, in which the price movements of the stocks are quite different. Generally, in a regime-switching model, the value of market modes are divided into a finite number of regimes. The key parameters, such as the bank interest rate, stocks appreciation rates, and volatility rates, will change according to the value of different market modes. Since the market state may change from one regime to another, both the nature of the regime and the change point should be estimated. If the market state process is modeled by a continuous time Markov chain with finite states, regime switching 
models are also referred to as Markov switching or Markov-modulated models in some literatures.

With time-varying parameters, regime switching models are obviously more realistic than constant parameter model to reflect the random market environment. As discussed in Neftci (1984), an appealing ability of these models is to account for the accumulating evidence that business cycles are asymmetric. Most of the studies indicate that regimeswitching models perform well in some sense, for example, Hardy (2001) used monthly data from the Standard and Poor's 500 and the Toronto Stock Exchange 300 indices to fit a regime switching lognormal model. In their paper, the fit of the regime switching model to the data was compared with other econometric models and she found that regimeswitching models provided a significant improvement over all the other models in the sense of maximizing the likelihood function. In a special case, if the data is in lognormal setting, the software "regime switching equity model workbook" developed by Hardy and her group which can be found on SOA web site can be applied directly, which greatly simplifies the application procedure of regime switching model.

Regime switching models are not new in statistics and economics, dating back to at least Quandt (1958), where regime regression models are investigated. Kim and Nelson (1999) gave a brief review of Markov switching models and presented comprehensive exposition of statistical methods for these models as well as many empirical studies. One influential work on the application of regime switching models is Hamilton (1989), where dynamic models with Markov switching between regimes are introduced as a tool for dealing with endogenous structural breaks. And after that, enormous empirical works about regime switching structure were done in many economical aspects, such as business cycle asymmetry, see Hamilton (1989), Lam (1990); the effects of oil prices on U.S. GDP 
growth, see Raymond and Rich (1997); labor market recruitment, see Storer (1996); government expenditure, see Rugemurcia (1995); and the level of merger and acquisition activity (Town, 1992). etc

However, it is recent years for the application of regime switching models in finance. Early works are done on option pricing, see Di Masi et al. (1994), Buffington and Elliott (2002), Boyle and Draviam (2007). After that, regime switching models are applied to many other aspects, such as Equity-linked life insurance pricing, see Hardy (2003); Bond Pricing, see Elliott and Siu (2008a); Portfolio selection, see Zhou and Yin (2003), Chen et al (2008), Elliott and Siu (2008b); Optimal dividend, Li and Lu (2006,2007), Sotomayor and Cadenillas (2008), Wei et al. (2009). etc.

In this paper, we extend the pension funding model in Gerber and Shiu (2003) to a regime switching model. Gerber and Shiu (2003) presented an elegant application of Geometric Brownian motion to asset and liability management. In their formulation, the asset value process $A(t)$ and liability value process $L(t)$ are modeled by correlated geometric Brownian motions, where the drift parameters and instantaneous standard deviations are modulated by the Markov chain. They considered a pension funding plan in which the asset value $A(t)$ is to be within a band bounded by $L_{1}(t)=\lambda_{1} L(t)$ and $L_{2}(t)=\lambda_{2} L(t)$ for some parameters $\lambda_{2}>\lambda_{1}>0$. The pension plan sponsor is asked to provide sufficient funds to guarantee the asset value $A(t)$ stays above the lower barrier $L_{1}(t)$ of the band. On the other hand, the amount by which the asset value exceeds the upper barrier $L_{2}(t)$ will be paid back to the sponsor. Then the original asset value $A(t)$ is replaced by the modified asset value $A^{m}(t)$. We adopt the same notation $A(t), L(t)$, $L_{1}(t)$ and $L_{2}(t)$ in our paper. Since our consideration is in a regime switching setting, all the parameters will be modulated by a Markov chain that may change from one regime to 
another, then the parameters sets of $A^{m}(t), L_{1}(t)$ and $L_{2}(t)$ will also change according to the Markov chain. A path of the regime switching version of these processes are illustrated in Figure 1, from which we can see clear change of the three processes before and after the change point $t=1$.

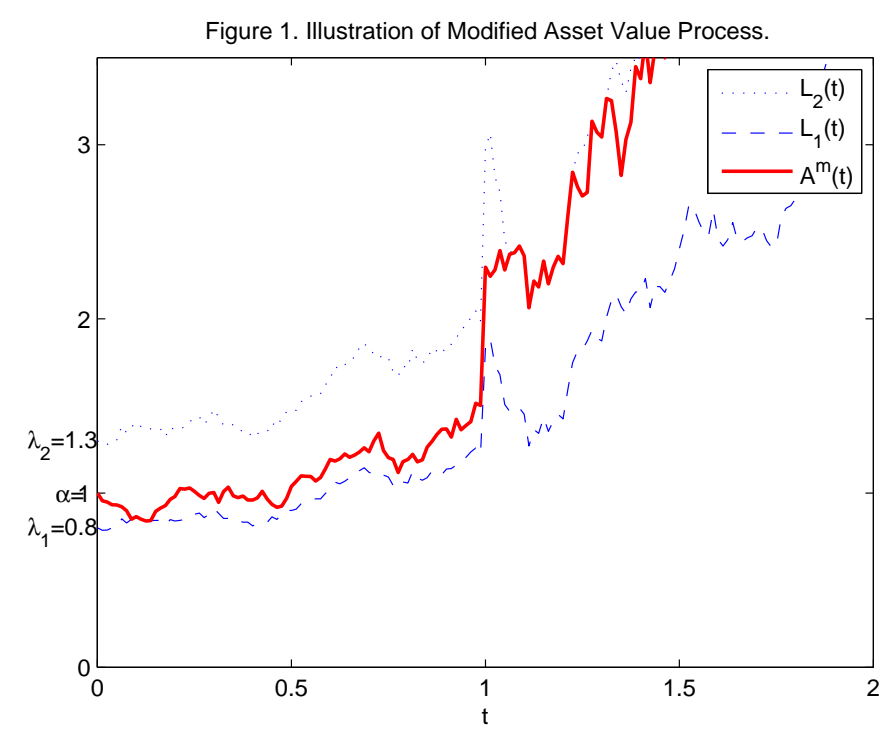

In this paper, we shall calculate $V_{1}\left(\alpha ; \lambda_{1}, \lambda_{2}, i_{0}\right)$, the expectation of the present value of the payments that have to be provided by the sponsor to guarantee that the asset is not below the lower level $L_{1}(t)$, and $V_{2}\left(\alpha ; \lambda_{1}, \lambda_{2}, i_{0}\right)$, the expectation of the present value of the "overflow" to maintain the upper level $L_{2}(t)$. Here $\alpha$ is the time zero asset value which belongs to $\left[\lambda_{1}, \lambda_{2}\right]$ and $i_{0}$ is the market mode when the pension plan is launched. By applying a differential equation approach, a system of second order differential equations indexed by market modes $i_{0} \in \mathcal{M}$ is derived for $V_{j}\left(\alpha ; \lambda_{1}, \lambda_{2}, i_{0}\right)$ for $j=1,2$. The equations will be solved by linear algebra method in Section 3. We give some numerical results in Section 4.1-4.2 to illustrate the effects of $\lambda_{1}$ and $\lambda_{2}$ and regime switching phenomenon. Finally, as an application of our barrier model for asset and liability value process, the optimal dividend problem will be studied in our numerical examples in Section 4.3.

The paper is organized as follows. Section 2 is the formulation of the model. In 
Section 3 , the differential equations for $V_{j}\left(\alpha ; \lambda_{1}, \lambda_{2}, i_{0}\right), j=1,2, i_{0} \in \mathcal{M}$ are derived and the solution method is presented. Section 4 provides some numerical examples to illustrate our results.

\section{The Model}

Throughout the paper, let $(\Omega, \mathcal{F}, P)$ be a fixed complete probability space and let $W(t)=$ $\left(W_{1}(t), W_{2}(t)\right)^{\prime}$ be a bivariate standard Brownian motion with constant correlation $\rho$. The market mode is divided into $d$ different regimes which are represented by the regime space $\mathcal{M}=\{1,2, \ldots, d\}$. Let $\beta_{t}$ be a continuous-time stationary Markov chain taking value in $\mathcal{M}$ such that $W(t)$ and $\beta_{t}$ are independent of each other. The Markov chain has a transition rate matrix $Q=\left(q_{i j}\right) \in \mathbb{R}^{d \times d}$ (conventionally called $Q$-matrix) and stationary transition probabilities

$$
p_{i j}(t)=\operatorname{Pr}\left(\beta_{t}=j \mid \beta_{0}=i\right), t \geq 0, i, j \in \mathcal{M} \text {. }
$$

As is implied in some empirical study, the market mode is seldom instantaneous state, see for example, Hardy (2001). That is, for each $i \in \mathcal{M}$, we assume $\left|q_{i i}\right|<+\infty$.

Due to the positive nature of asset and liability values, we model them by geometric Brownian motions of the form

$$
\begin{aligned}
& A(t)=\alpha e^{X(t)}, \\
& L(t)=\lambda e^{Y(t)},
\end{aligned}
$$


with $X(t)$ and $Y(t)$ satisfying the following stochastic differential equation:

$$
\begin{array}{ll}
\mathrm{d} X(t)=\mu_{X}\left(\beta_{t}\right) \mathrm{d} t+\sigma_{X}\left(\beta_{t}\right) \mathrm{d} W_{1}(t), & X(0)=0 \\
\mathrm{~d} Y(t)=\mu_{Y}\left(\beta_{t}\right) \mathrm{d} t+\sigma_{Y}\left(\beta_{t}\right) \mathrm{d} W_{2}(t), & Y(0)=0
\end{array}
$$

where $\mu_{X}(i)$ and $\mu_{Y}(i)$ for $i \in \mathcal{M}$ are given as the drift parameters corresponding to different market modes, $\sigma_{X}(i)$ and $\sigma_{Y}(i)$ are corresponding instantaneous standard deviations. We shall study the case where $A(t)$ is bounded by upper and lower multiples of $L(t)$, that is,

$$
L_{j}(t)=\lambda_{j} e^{Y(t)}, \quad j=1,2
$$

with $0<\lambda_{1}<\lambda_{2}$. It is assumed that between the barriers $L_{1}(t)$ and $L_{2}(t)$, the modified asset value process $A^{m}(t)$ has the same instantaneous rate of return as the original asset value process $A(t)$. Figure 1 plots $L_{2}(t), A^{m}(t)$ and $L_{1}(t)$ from above to below with the assumption that the market mode switches from "bearish" to "bullish" at time 1, which can be seen clearly from the paths of three processes.

Since $\mathrm{E}\left[A(t) \mid \mathcal{F}_{t}\right]=\alpha e^{\int_{0}^{t}\left[\mu_{X}\left(\beta_{s}\right)+\frac{1}{2} \sigma_{X}\left(\beta_{s}\right)^{2}\right] \mathrm{d} s}$, we introduce the asset growth rate corresponding to market mode $i \in \mathcal{M}$ as

$$
\delta_{X}(i)=\mu_{X}(i)+\frac{1}{2} \sigma_{X}(i)^{2}
$$

Similarly, the liability growth rates are defined as

$$
\delta_{Y}(i)=\mu_{Y}(i)+\frac{1}{2} \sigma_{Y}(i)^{2}, \quad i \in \mathcal{M}
$$

The force of interest $\delta(i)$ is also assumed to be affected by market mode $i \in \mathcal{M}$. For 
notational simplicity, we introduce

$$
\sigma(i)^{2}=\sigma_{X}(i)^{2}-2 \rho \sigma_{X}(i) \sigma_{Y}(i)+\sigma_{Y}(i)^{2}, \quad i \in \mathcal{M}
$$

Let $\tau$ be the first time when the asset value reaches one of the two barriers, then

$$
\tau=\min \left\{t \geq 0 \mid A(t)=L_{1}(t) \text { or } A(t)=L_{2}(t)\right\}
$$

With the time zero asset value $\alpha \in\left[\lambda_{1}, \lambda_{2}\right]$ and market mode $\beta_{0}=i_{0}$, let $V_{1}\left(\alpha ; \lambda_{1}, \lambda_{2}, i_{0}\right)$ be the expectation of the present value of the payments that have to be provided by the sponsor to guarantee that the asset is not below the lower level $L_{1}(t)$. If we treat time $\tau$ as the new orientation time for another pension plan, then the starting condition become $A(\tau), L_{1}(\tau), L_{1}(\tau)$ and $\beta_{\tau}$. Since no barrier is reached before time $\tau$, no payment is made during the time period $[0, \tau)$, which results in the expected present value of $V_{1}\left(A(\tau) ; L_{1}(\tau), L_{2}(\tau), \beta_{\tau}\right)$ is just $V_{1}\left(\alpha ; \lambda_{1}, \lambda_{2}, i_{0}\right)$ given the initial condition $A(0)=$ $\alpha, L_{1}(0)=\lambda_{1}, L_{2}(0)=\lambda_{2}$ and $\beta_{0}=i_{0}$. This argument can be written as follows,

$$
\begin{aligned}
& V_{1}\left(\alpha ; \lambda_{1}, \lambda_{2}, i_{0}\right)= \\
& \mathrm{E}\left[e^{-\int_{0}^{\tau} \delta\left(\beta_{s}\right) \mathrm{d} s} V_{1}\left(A(\tau) ; L_{1}(\tau), L_{2}(\tau), \beta_{\tau}\right) \mid A(0)=\alpha, L_{1}(0)=\lambda_{1}, L_{2}(0)=\lambda_{2}, \beta_{0}=i_{0}\right]
\end{aligned}
$$

Similarly, let $V_{2}\left(\alpha ; \lambda_{1}, \lambda_{2}, i_{0}\right)$ be the expectation of the present value of the "overflow" to maintain the upper level $L_{2}(t)$, then

$$
\begin{aligned}
& V_{2}\left(\alpha ; \lambda_{1}, \lambda_{2}, i_{0}\right)= \\
& \mathrm{E}\left[e^{-\int_{0}^{\tau} \delta\left(\beta_{s}\right) \mathrm{d} s} V_{2}\left(A(\tau) ; L_{1}(\tau), L_{2}(\tau), \beta_{\tau}\right) \mid A(0)=\alpha, L_{1}(0)=\lambda_{1}, L_{2}(0)=\lambda_{2}, \beta_{0}=i_{0}\right]
\end{aligned}
$$




\section{The Calculation of $V_{j}\left(\alpha ; \lambda_{1}, \lambda_{2}, i_{0}\right)$ for $j=1,2$}

In this section, following Gerber and Shiu (2003), we apply a differential equation approach to calculate $V_{j}\left(\alpha ; \lambda_{1}, \lambda_{2}, i_{0}\right)$ for $j=1,2$. Note that $A(t)$ and $L(t)$ are geometric Brownian motions, then the functions $V_{j}\left(\alpha ; \lambda_{1}, \lambda_{2}, i_{0}\right)$ for $j=1,2$ are homogeneous of degree 1 with respect to $\alpha, \lambda_{1}$ and $\lambda_{2}$. That is, for each $z>0$, we have

$$
V_{j}\left(z \alpha ; z \lambda_{1}, z \lambda_{2}, i_{0}\right)=z V_{j}\left(\alpha ; \lambda_{1}, \lambda_{2}, i_{0}\right), \quad j=1,2
$$

Based on this homogeneous property and the expressions (2.11) and (2.12), we see in the latter part of this section that $V_{1}\left(\alpha ; \lambda_{1}, \lambda_{2}, i_{0}\right)$ and $V_{2}\left(\alpha ; \lambda_{1}, \lambda_{2}, i_{0}\right)$ follow the same differential equation. The main difference between them is boundary conditions,

$$
\begin{aligned}
& V_{1}^{\prime}\left(\lambda_{1} ; \lambda_{1}, \lambda_{2}, i_{0}\right)=-1, \\
& V_{1}^{\prime}\left(\lambda_{2} ; \lambda_{1}, \lambda_{2}, i_{0}\right)=0 \\
& V_{2}^{\prime}\left(\lambda_{1} ; \lambda_{1}, \lambda_{2}, i_{0}\right)=0 \\
& V_{2}^{\prime}\left(\lambda_{2} ; \lambda_{1}, \lambda_{2}, i_{0}\right)=1
\end{aligned}
$$

for each $i_{0} \in \mathcal{M}$. Conditions (3.2)-(3.5) are derived by heuristic justification as follows. If the time zero asset value $\alpha$ is close to $\lambda_{1}$, the first time $\tau$ for the asset value process to reach one of the two barriers is close to 0 . Then in an infinitesimal time interval, the market mode remains at $i_{0}$, and when $\alpha$ falls below $\lambda_{1}$, the amount $\left|\alpha-\lambda_{1}\right|=-\left(\alpha-\lambda_{1}\right)$ has to be provided to guarantee the lower bound $\lambda_{1}$, which explains (3.2). On the other hand, since $\lambda_{1}<\lambda_{2}$, no dividend that has to be paid to guarantee the upper bound $\lambda_{2}$, which explains (3.4). Similarly, if the time zero asset value $\alpha$ is close to $\lambda_{2}$, the time $\tau$ is 
close to 0 and the market mode remains at $i_{0}$. Then in an infinitesimal time interval, when $\alpha$ overflows $\lambda_{2}$, the amount $\left|\alpha-\lambda_{2}\right|=\alpha-\lambda_{2}$ has to be paid out as dividend to guarantee the upper bound $\lambda_{2}$, which explains (3.5). For the similar reason as (3.4), condition (3.3) can be explained.

Based on the above observation, for notational simplicity, $V\left(\alpha ; \lambda_{1}, \lambda_{2}, i_{0}\right)$ can be either $V_{1}\left(\alpha ; \lambda_{1}, \lambda_{2}, i_{0}\right)$ or $V_{2}\left(\alpha ; \lambda_{1}, \lambda_{2}, i_{0}\right)$ in the following of the paper.

Consider the infinitesimal time interval from 0 to $\mathrm{d} t$. As stated in Section 2, the time zero market mode $i_{0}$ is not instantaneous state, then the force of interest $\delta\left(\beta_{t}\right)$ remains at $\delta\left(i_{0}\right)$ during 0 to $\mathrm{d} t$. With $\lambda_{1}<\alpha<\lambda_{2}$, we have $\tau>\mathrm{d} t$. In the same spirit of $(2.11)$ and (2.12), since no payment is made during [0,dt), we have

$$
\begin{aligned}
& V\left(\alpha ; \lambda_{1}, \lambda_{2}, i_{0}\right) \\
& =\mathrm{E}\left[e^{-\delta\left(i_{0}\right) \mathrm{d} t} V\left(A(\mathrm{~d} t) ; L_{1}(\mathrm{~d} t), L_{2}(\mathrm{~d} t), \beta_{\mathrm{d} t}\right) \mid A(0)=\alpha, L_{1}(0)=\lambda_{1}, L_{2}(0)=\lambda_{2}, \beta_{0}=i_{0}\right]
\end{aligned}
$$

Because of homogeneous property of $V$,

$$
V\left(A(\mathrm{~d} t) ; L_{1}(\mathrm{~d} t), L_{2}(\mathrm{~d} t), \beta_{\mathrm{d} t}\right)=e^{Y(\mathrm{~d} t)} V\left(\alpha e^{X(\mathrm{~d} t)-Y(\mathrm{~d} t)} ; \lambda_{1}, \lambda_{2}, \beta_{\mathrm{d} t}\right) .
$$

Neglecting terms whose expectation is of order smaller than $\mathrm{d} t$, we can write

$$
e^{-\delta\left(i_{0}\right) \mathrm{d} t+Y(\mathrm{~d} t)}=1-\delta\left(i_{0}\right) \mathrm{d} t+Y(\mathrm{~d} t)+\frac{1}{2}[Y(\mathrm{~d} t)]^{2}
$$


and

$$
\begin{aligned}
& V\left(\alpha e^{X(\mathrm{~d} t)-Y(\mathrm{~d} t)} ; \lambda_{1}, \lambda_{2}, \beta_{\mathrm{d} t}\right) \\
= & V\left(\alpha ; \lambda_{1}, \lambda_{2}, \beta_{\mathrm{d} t}\right)+V^{\prime}\left(\alpha ; \lambda_{1}, \lambda_{2}, \beta_{\mathrm{d} t}\right) \alpha\left\{[X(\mathrm{~d} t)-Y(\mathrm{~d} t)]+\frac{1}{2}[X(\mathrm{~d} t)-Y(\mathrm{~d} t)]^{2}\right\} \\
& \quad+\frac{1}{2} V^{\prime \prime}\left(\alpha ; \lambda_{1}, \lambda_{2}, \beta_{\mathrm{d} t}\right) \alpha^{2}[X(\mathrm{~d} t)-Y(\mathrm{~d} t)]^{2} \\
= & V\left(\alpha ; \lambda_{1}, \lambda_{2}, i_{0}\right)+\sum_{k=1}^{d} q_{i_{0} k} V\left(\alpha ; \lambda_{1}, \lambda_{2}, k\right) \mathrm{d} t \\
& +\left[V^{\prime}\left(\alpha ; \lambda_{1}, \lambda_{2}, i_{0}\right)+\sum_{k=1}^{d} q_{i_{0} k} V^{\prime}\left(\alpha ; \lambda_{1}, \lambda_{2}, k\right) \mathrm{d} t\right] \alpha\left\{[X(\mathrm{~d} t)-Y(\mathrm{~d} t)]+\frac{1}{2}[X(\mathrm{~d} t)-Y(\mathrm{~d} t)]^{2}\right\} \\
& +\frac{1}{2}\left[V^{\prime \prime}\left(\alpha ; \lambda_{1}, \lambda_{2}, i_{0}\right)+\sum_{k=1}^{d} q_{i_{0} k} V^{\prime \prime}\left(\alpha ; \lambda_{1}, \lambda_{2}, k\right) \mathrm{d} t\right] \alpha^{2}[X(\mathrm{~d} t)-Y(\mathrm{~d} t)]^{2} .
\end{aligned}
$$

The product of $(3.8)$ and (3.9) is

$$
\begin{aligned}
& e^{-\delta\left(i_{0}\right) \mathrm{d} t+Y(\mathrm{~d} t)} V\left(\alpha e^{X(\mathrm{~d} t)-Y(\mathrm{~d} t)} ; \lambda_{1}, \lambda_{2}, \beta_{\mathrm{d} t}\right) \\
= & {\left[V\left(\alpha ; \lambda_{1}, \lambda_{2}, i_{0}\right)+\sum_{k=1}^{d} q_{i_{0}} V\left(\alpha ; \lambda_{1}, \lambda_{2}, k\right) \mathrm{d} t\right]\left\{1-\delta\left(i_{0}\right) \mathrm{d} t+Y(\mathrm{~d} t)+\frac{1}{2}[Y(\mathrm{~d} t)]^{2}\right\} } \\
& +V^{\prime}\left(\alpha ; \lambda_{1}, \lambda_{2}, i_{0}\right) \alpha\left\{[X(\mathrm{~d} t)-Y(\mathrm{~d} t)]+\frac{1}{2}[X(\mathrm{~d} t)-Y(\mathrm{~d} t)]^{2}+Y(\mathrm{~d} t)[X(\mathrm{~d} t)-Y(\mathrm{~d} t)]\right\} \\
& +\frac{1}{2} \alpha^{2} V^{\prime \prime}\left(\alpha ; \lambda_{1}, \lambda_{2}, i_{0}\right)[X(\mathrm{~d} t)-Y(\mathrm{~d} t)]^{2} .
\end{aligned}
$$

By (3.6) and remembering that $W(t)$ and $\beta_{t}$ are independent of each other, we take expectation to (3.10) to derive

$$
\begin{aligned}
& V\left(\alpha ; \lambda_{1}, \lambda_{2}, i_{0}\right) \\
& =V\left(\alpha ; \lambda_{1}, \lambda_{2}, i_{0}\right)\left[1-\delta\left(i_{0}\right) \mathrm{d} t+\delta_{Y}\left(i_{0}\right) \mathrm{d} t\right]+\sum_{k=1}^{d} q_{i_{0} k} V\left(\alpha ; \lambda_{1}, \lambda_{2}, k\right) \mathrm{d} t \\
& \quad+V^{\prime}\left(\alpha ; \lambda_{1}, \lambda_{2}, i_{0}\right) \alpha\left[\delta_{X}\left(i_{0}\right)+\delta_{Y}\left(i_{0}\right)\right] \mathrm{d} t+\frac{1}{2} V^{\prime \prime}\left(\alpha ; \lambda_{1}, \lambda_{2}, i_{0}\right) \alpha^{2} \sigma^{2}\left(i_{0}\right) \mathrm{d} t
\end{aligned}
$$

where $\delta_{X}\left(i_{0}\right), \delta_{Y}\left(i_{0}\right)$ and $\sigma\left(i_{0}\right)$ are defined by (2.7), (2.8) and (2.9) for $i=i_{0} \in \mathcal{M}$, 
respectively. Subtracting $V\left(\alpha ; \lambda_{1}, \lambda_{2}, i_{0}\right)$ on both sides and canceling $\mathrm{d} t$ in (3.11), we have

$$
\begin{gathered}
{\left[\delta_{Y}\left(i_{0}\right)-\delta\left(i_{0}\right)\right] V\left(\alpha ; \lambda_{1}, \lambda_{2}, i_{0}\right)+\alpha\left[\delta_{X}\left(i_{0}\right)-\delta_{Y}\left(i_{0}\right)\right] V^{\prime}\left(\alpha ; \lambda_{1}, \lambda_{2}, i_{0}\right)} \\
+\frac{1}{2} \alpha^{2} \sigma^{2}\left(i_{0}\right) V^{\prime \prime}\left(\alpha ; \lambda_{1}, \lambda_{2}, i_{0}\right)+\sum_{k=1}^{d} q_{i_{0}} V\left(\alpha ; \lambda_{1}, \lambda_{2}, k\right)=0
\end{gathered}
$$

which is a system of ordinary differential equations with respect to $\alpha$ indexed by $i_{0} \in \mathcal{M}$.

In the case that there is no regime-switching phenomenon, that is, $q_{i j}=0$ for all $i, j \in \mathcal{M}$, equation (3.12) turns out to be

$$
\begin{aligned}
{\left[\delta_{Y}\left(i_{0}\right)-\delta\left(i_{0}\right)\right] V\left(\alpha ; \lambda_{1}, \lambda_{2}, i_{0}\right)+\alpha\left[\delta_{X}\left(i_{0}\right)-\delta_{Y}\left(i_{0}\right)\right] V^{\prime}\left(\alpha ; \lambda_{1}, \lambda_{2}, i_{0}\right) } & \\
& +\frac{1}{2} \alpha^{2} \sigma^{2}\left(i_{0}\right) V^{\prime \prime}\left(\alpha ; \lambda_{1}, \lambda_{2}, i_{0}\right)=0
\end{aligned}
$$

which is consistent with the result in Gerber and Shiu (2003).

Within complex domain, the general solutions for (3.12) and (3.13) always exist and unique under the boundary conditions (3.2) to (3.5). To guarantee a real-valued solution of (3.13), Gerber and Shiu (2003) introduced an assumption

$$
\delta_{Y}<\delta
$$

However, for a system of ordinary differential equations (3.12), it is not easy to find such neat and simple condition as (3.14) to guarantee real-valued solutions. Even if we can find some conditions, they may not be applicable in practice. To solve (3.12), we first notice that it is in the same type of a system of differential equations

$$
a_{i} \alpha^{2} f_{i}^{\prime \prime}(\alpha)+b_{i} \alpha f_{i}^{\prime}(\alpha)+c_{i} f_{i}(\alpha)+\sum_{j \neq i} c_{i j} f_{j}(\alpha)=0, i, j \in \mathcal{M}
$$


where $f_{i}(\alpha)$ is a function with respect to $\alpha$ for $i \in \mathcal{M}$ and $a_{i}, b_{i}$ and $c_{i j}$ are constant coefficients corresponding to $i, j \in \mathcal{M}$. Since $\alpha$ is the initial asset value which should be positive, let $\alpha=e^{s}>0$ for $s \in \mathbb{R}$ and define $g_{i}(s):=f_{i}\left(e^{s}\right)$ for $i \in \mathcal{M}$, then (3.15) is transformed into

$$
a_{i} g_{i}^{\prime \prime}(s)+\left(b_{i}-a_{i}\right) g_{i}^{\prime}(s)+c_{i} g_{i}(s)+\sum_{j \neq i} c_{i j} g_{j}(s)=0, i, j \in \mathcal{M}
$$

which is a system of second-order linear differential equations. To solve (3.16), let

$$
h_{i}(s):=g_{i}^{\prime}(s), i \in \mathcal{M}
$$

and

$$
H(s)=\left(g_{1}(s), \ldots, g_{d}(s), h_{1}(s), \ldots, h_{d}(s)\right)^{\prime} .
$$

Under the condition that for each $i \in \mathcal{M}$,

$$
a_{i} \neq 0
$$

and based on (3.16) and (3.17), an equivalent system of linear differential equations to (3.15) can be derived

$$
\frac{\mathrm{d} H(s)}{\mathrm{d} s}=A H(s)
$$


where $A$ is a $2 d \times 2 d$ square matrix defined as

$$
A=-\left(\begin{array}{cccccccc}
0 & 0 & \cdots & 0 & -1 & 0 & \cdots & 0 \\
0 & 0 & \cdots & 0 & 0 & -1 & \cdots & 0 \\
\vdots & \vdots & \ddots & \vdots & \vdots & \vdots & \ddots & \vdots \\
0 & 0 & \cdots & 0 & 0 & 0 & \cdots & -1 \\
\frac{c_{11}}{a_{1}} & \frac{c_{12}}{a_{1}} & \cdots & \frac{c_{1 d}}{a_{1}} & \frac{b_{1}}{a_{1}}-1 & 0 & \cdots & 0 \\
\frac{c_{21}}{a_{2}} & \frac{c_{22}}{a_{2}} & \cdots & \frac{c_{2 d}}{a_{2}} & 0 & \frac{b_{2}}{a_{2}}-1 & \cdots & 0 \\
\vdots & \vdots & \ddots & \vdots & \vdots & \vdots & \ddots & \vdots \\
\frac{c_{d 1}}{a_{d}} & \frac{c_{d 2}}{a_{d}} & \cdots & \frac{c_{d d}}{a_{d}} & 0 & 0 & \cdots & \frac{b_{d}}{a_{d}}-1
\end{array}\right) .
$$

Note that (3.20) is a homogeneous first-order linear differential equation system, the closed-form solutions of which can be derived by investigating the eigenvalues and eigenvectors of matrix $A$. We also note that if $d=1$, that is, in the case of no regime-switching phenomenon, we have

$$
A=-\left(\begin{array}{cc}
0 & -1 \\
\frac{c_{11}}{a_{1}} & \frac{b_{1}}{a_{1}}-1
\end{array}\right) \text {. }
$$

whose characteristic function corresponding to $\theta$ is

$$
\theta^{2}+\frac{b_{1}-a_{1}}{a_{1}} \theta+\frac{c_{11}}{a_{1}}=0 .
$$

Plugging the original coefficients in (3.12) into the above equation, we have

$$
\frac{1}{2} \sigma^{2}\left(i_{0}\right) \theta(\theta-1)+\left[\delta_{X}\left(i_{0}\right)-\delta_{Y}\left(i_{0}\right)\right] \theta+\delta_{Y}\left(i_{0}\right)-\delta\left(i_{0}\right)=0
$$

which admits two different real-valued roots under the condition (3.14). However, in 
the case that $d \geq 2$, it is not easy to add constrains to all the coefficients to guarantee real-valued eigenvalues for matrix $A$. This is also the reason that (3.12) may not admit real-valued solutions. Based upon a lot of numerical examples, we find that real-valued solutions exist in many cases, which are not merely confined to the condition (3.14). In the next section, we shall show by examples how this solving procedure works and illustrate the effects of some main parameters on the solution.

\section{Numerical Result}

In this section we give several examples in three subsections to illustrate our result. In all the following examples, the market mode is divided as "bullish" and "bearish" two regimes, which corresponds to regime 1 and regime 2 in $\mathcal{M}=\{1,2\}$, respectively. $V_{j}\left(\alpha ; \lambda_{1}, \lambda_{2}, i_{0}\right)$ for $j=1,2$ are calculated based on the solving method for (3.12), which is roughly stated in the latter part of the Section 3. Specifically, it follows from the equivalence of (3.12) and (3.15) that

$$
\begin{aligned}
& a_{i}=\frac{1}{2} \sigma^{2}(i), \\
& b_{i}=\delta_{X}(i)-\delta_{Y}(i), \\
& c_{i i}=\delta_{Y}(i)-\delta(i)+q_{i i}, \\
& c_{i j}=q_{i j}, j \neq i
\end{aligned}
$$

with $i, j=1,2$. Plugging all the parameters above into matrix A (see (3.21)) and calculating the corresponding eigenvalues $\theta_{i}$ (assume they are real-valued) and eigenvectors $\eta_{i}$ for $i=1, \ldots, 4$, we have

$$
H(s)=\sum_{i=1}^{4} C_{i} e^{\theta_{i} s} \eta_{i}
$$


where constants $C_{i}$ can be calculated through conditions (3.2)-(3.5). Remember that $g_{1}(s)$ and $g_{2}(s)$ are the first two-dimension of $H(s)$, and $\alpha=e^{s}$, then the original solution for (3.15) corresponding to $\alpha$ can be solved.

\subsection{The effect of barriers $\lambda_{1}$ and $\lambda_{2}$}

Generally, the barrier $\lambda_{1}$ and $\lambda_{2}$ are given beforehand in a pension funding plan. Heuristically, whatever the market mode would be when the plan is launched, the larger the $\lambda_{1}$, the more amount of money needs to be provided by the sponsor to guarantee the lower bound, that is, the larger the $V_{1}\left(\alpha ; \lambda_{1}, \lambda_{2}, i\right)$ for $i=1,2$. Meanwhile, if $\lambda_{2}$ maintains unchanged, since the sponsor injects more funds into the plan, it is more likely to pay out money to the sponsor than before, that is, the larger of $V_{2}\left(\alpha ; \lambda_{1}, \lambda_{2}, i\right)$ for $i=1,2$. Similarly, the less the $\lambda_{2}$, the larger amount will be paid to the sponsor, that is, the greater of $V_{2}\left(\alpha ; \lambda_{1}, \lambda_{2}, i\right)$ for $i=1,2$. Meanwhile, if $\lambda_{1}$ maintains unchanged, since more money is paid back to the sponsor, it is more "tough" for the value process to stay above $\lambda_{1}$, that is, more funds is needed to guarantee the lower bound, which results in a larger $V_{1}\left(\alpha ; \lambda_{1}, \lambda_{2}, i\right)$ for $i=1,2$. This argument can be illustrated by the following example.

Example 4.1. Let $\rho=0.3, \lambda_{1}=0.9$ and $\lambda_{2}=1.2$. All the other parameters that related to regime switching are listed in Table 1.

Table 1

\begin{tabular}{|c|c|c|c|c|c|c|}
\hline & $\delta$ & $\mu_{X}$ & $\sigma_{X}^{2}$ & $\mu_{Y}$ & $\sigma_{Y}^{2}$ & transition rate \\
\hline regime 1 (bullish) & 0.3 & 0.4 & 0.18 & 0.2 & 0.09 & $q_{12}=0.4$ \\
\hline regime 2 (bearish) & 0.1 & 0.12 & 0.09 & 0.06 & 0.04 & $q_{21}=0.6$ \\
\hline
\end{tabular}

Calculating based on (2.7), (2.8) and (2.9), the defined parameters are 
Table 2

\begin{tabular}{|c|c|c|c|}
\hline & $\delta_{X}$ & $\delta_{Y}$ & $\sigma$ \\
\hline regime 1 (bullish) & 0.49 & 0.245 & 0.1936 \\
\hline regime 2 (bearish) & 0.165 & 0.08 & 0.08 \\
\hline
\end{tabular}

Based on equations (4.1), plugging all the parameters above into matrix $\mathrm{A}$ in (3.21), the corresponding eigenvalues and eigenvectors can be derived. Following the steps stated at the beginning of this Section, we have

$$
\begin{aligned}
& V_{1}(\alpha ; 0.9,1.2,1)=-0.0022 \alpha^{3.7105}+0.0056 \alpha^{-4.7059}+3.7802 \alpha^{0.3187}+1.0179 \alpha^{-1.6624}, \\
& V_{2}(\alpha ; 0.9,1.2,1)=-0.0028 \alpha^{3.7105}+0.0070 \alpha^{-4.7059}+8.1545 \alpha^{0.3187}+1.2381 \alpha^{-1.6624}, \\
& V_{1}(\alpha ; 0.9,1.2,2)=0.0078 \alpha^{3.7105}-0.0139 \alpha^{-4.7059}+3.7607 \alpha^{0.3187}+1.1039 \alpha^{-1.6624} \\
& V_{2}(\alpha ; 0.9,1.2,2)=0.0101 \alpha^{3.7105}-0.0173 \alpha^{-4.7059}+8.1124 \alpha^{0.3187}+1.3426 \alpha^{-1.6624}
\end{aligned}
$$

which are illustrated by Figure 2. Since larger $\alpha$ will result in less supplementary fund to guarantee the lower barrier $L_{1}(t)$ and more likely to pay back the "overflow" of $L_{2}(t)$ to the sponsor, which are equivalent to the less $V_{1}\left(\alpha ; \lambda_{1}, \lambda_{2}, i_{0}\right)$ and the larger $V_{2}\left(\alpha ; \lambda_{1}, \lambda_{2}, i_{0}\right)$. This prospective judgement can be verified by the decreasing trend of $V_{1}\left(\alpha ; 0.9,1.2, i_{0}\right)$ and the increasing trend of $V_{2}\left(\alpha ; 0.9,1.2, i_{0}\right)$ for $i_{0}=1,2$ in Figure 2.

As for the effects of different barriers on the result, we plot in Figure 3 four cases for comparison. In Figure 3, we take the subplots (I) and (II) as reference case, in which $\lambda_{1}=0.9$ and $\lambda_{2}=1.2$. All of the four subplots in each column have the same starting market mode $i_{0}$ and the same initial asset values, which make the comparison more clear. The upper lines in all of the eight subplots are $V_{2}\left(\alpha ; \lambda_{1}, \lambda_{2}, i_{0}\right)$ and the lower ones are $V_{1}\left(\alpha ; \lambda_{1}, \lambda_{2}, i_{0}\right)$. In (III) and (IV), we adjust $\lambda_{1}$ to 0.95 (larger) with $\lambda_{2}$ unchanged, then 

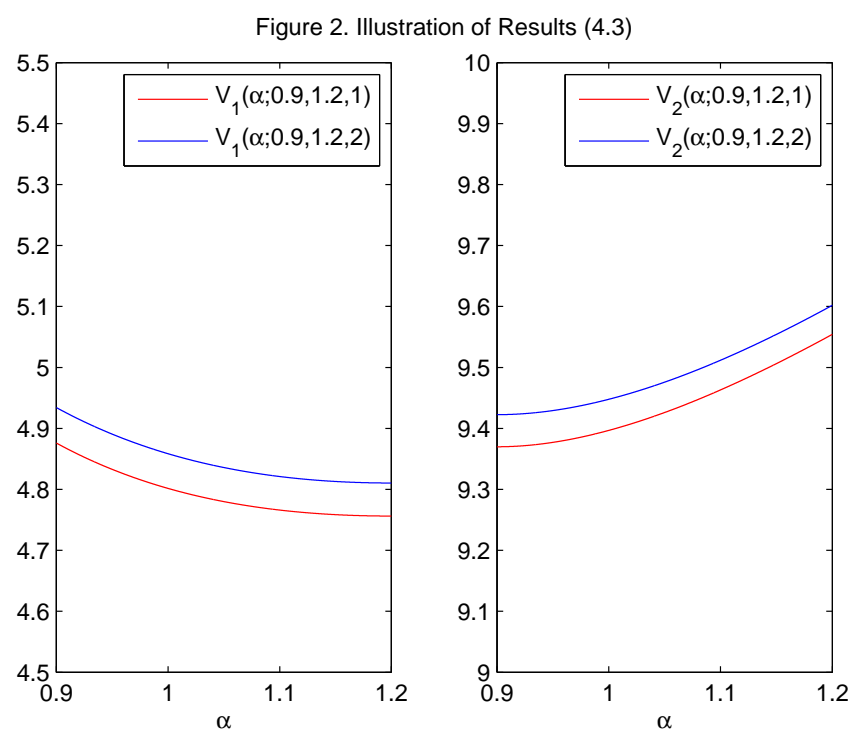

Figure 3. Illustration of Barriers Effect for Example 1
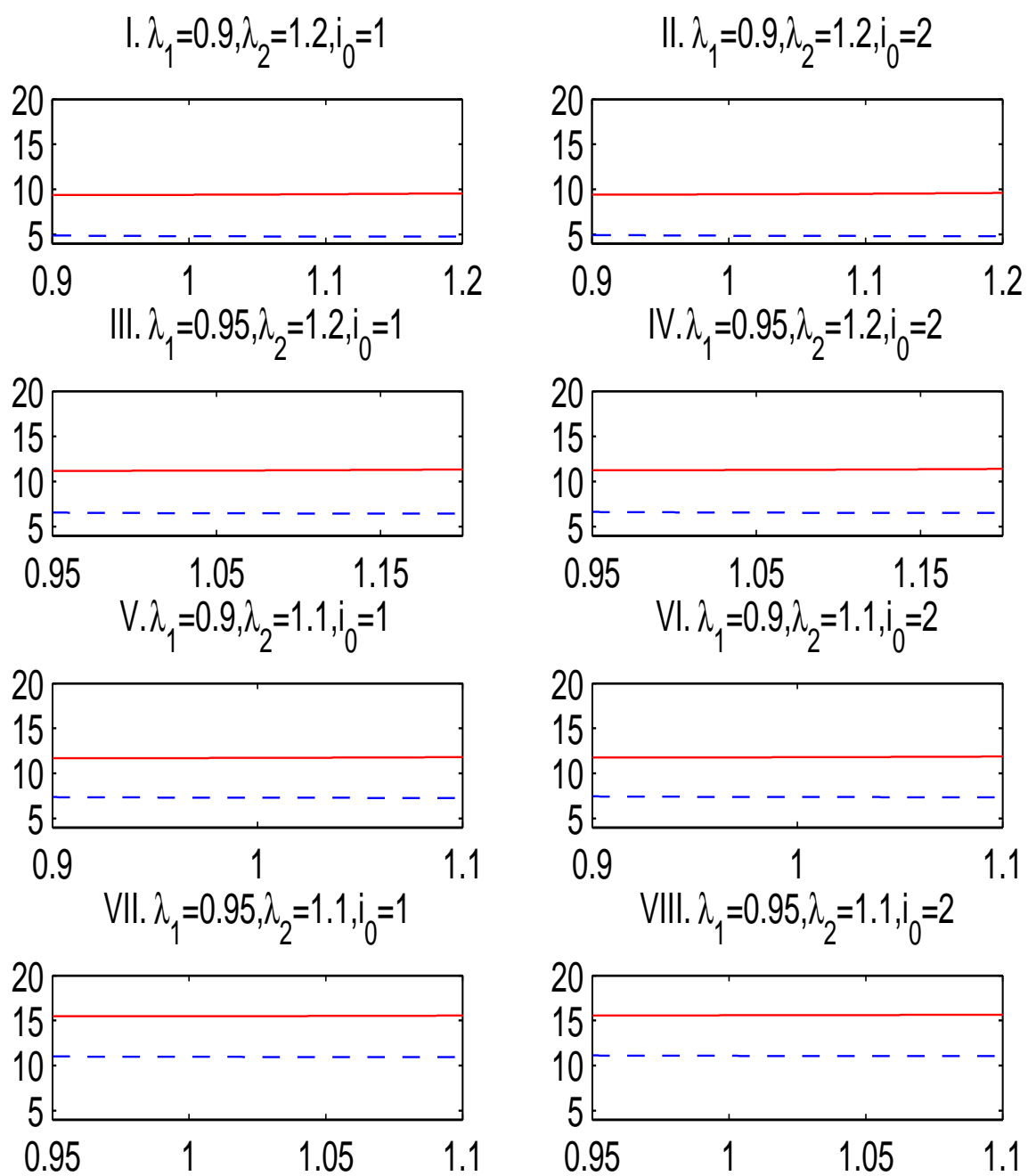

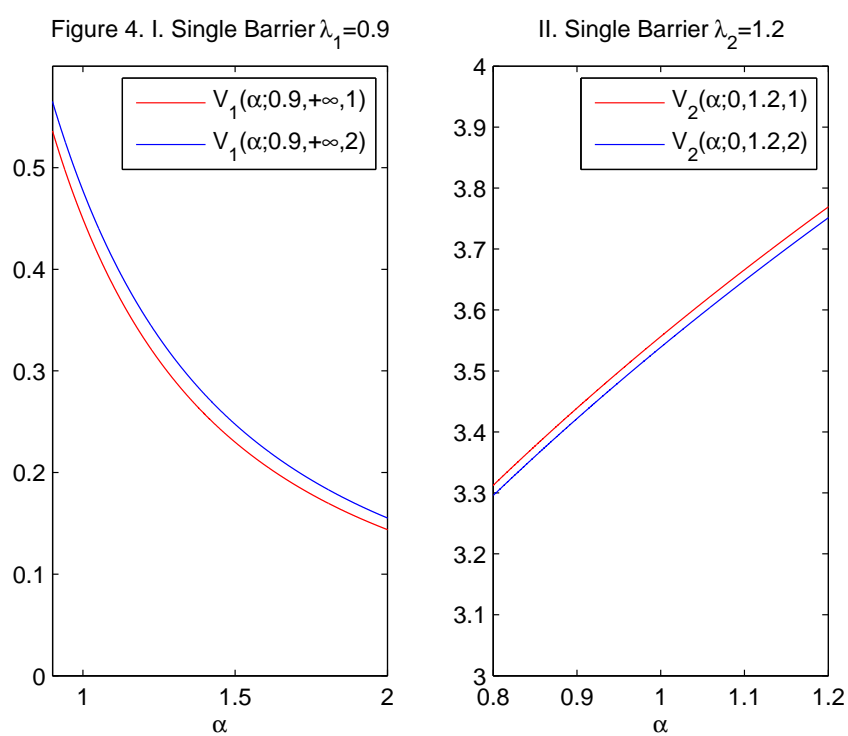

we find both $V_{1}\left(\alpha ; \lambda_{1}, \lambda_{2}, i_{0}\right)$ and $V_{2}\left(\alpha ; \lambda_{1}, \lambda_{2}, i_{0}\right)$ become larger than in (I) and (II). In (V) and (VI), we adjust $\lambda_{2}$ to 1.1 (smaller) with $\lambda_{1}$ unchanged, which also results in larger values of both $V_{1}\left(\alpha ; \lambda_{1}, \lambda_{2}, i_{0}\right)$ and $V_{2}\left(\alpha ; \lambda_{1}, \lambda_{2}, i_{0}\right)$. In (VII) and (VIII), both $\lambda_{1}$ and $\lambda_{2}$ are adjusted, which results in even larger values of $V_{1}\left(\alpha ; \lambda_{1}, \lambda_{2}, i_{0}\right)$ and $V_{2}\left(\alpha ; \lambda_{1}, \lambda_{2}, i_{0}\right)$ than the former three cases. These observations illustrate our argument appropriately.

Remark 4.1. An interesting special case in Example 1 is there is no need to pay the dividend (to the sponsor), which can be seen as the limiting case when $\lambda_{2}$ goes to $+\infty$. Then $V_{2}\left(\alpha ; \lambda_{1}, \lambda_{2}, i_{0}\right)$ for $i_{0}=1,2$ go to 0 , and the expected present payment to guarantee $\lambda_{1}=0.9$ in two regimes are plotted in (I) in Figure 4. Note that $\alpha \in[0.9,+\infty)$ in this case. Similarly, if no further support is needed from the sponsor after the pension plan is launched, let $\lambda_{1}=0$, then $\alpha \in(0,1.2]$ and $V_{1}\left(\alpha ; \lambda_{1}, \lambda_{2}, 1\right)=V_{1}\left(\alpha ; \lambda_{1}, \lambda_{2}, 2\right)=0$, the corresponding expected dividend is plotted in (II) in Figure 4. 


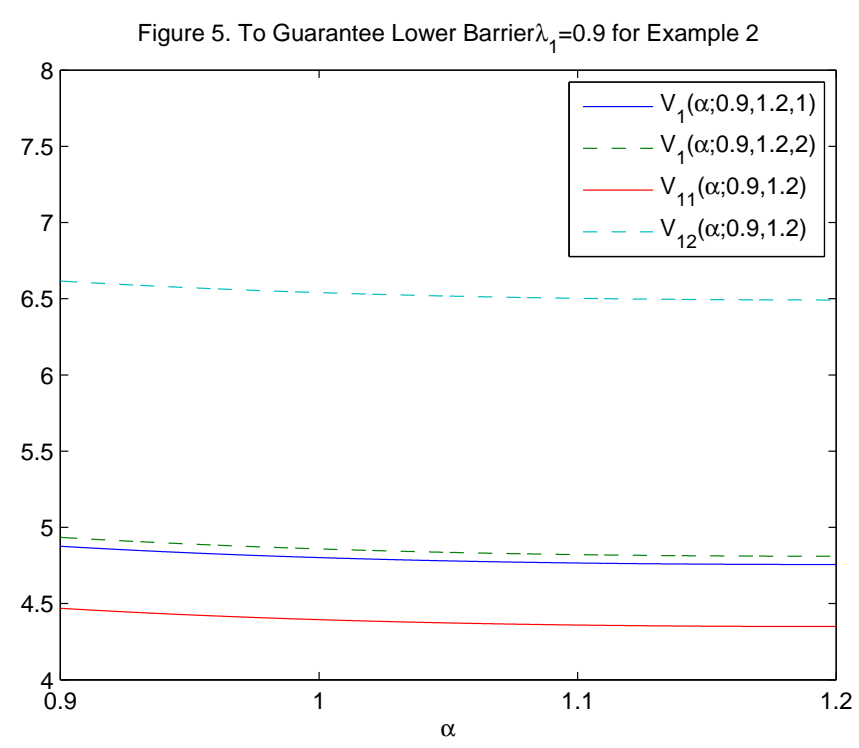

\subsection{The effects of regime switching}

In this subsection, we are concerned with the regime switching aspect of the model. With all the parameters the same as Example 1, we study in Example 2 the present expected value to guarantee the lower barrier $\lambda_{1}=0.9$ in purely bullish case and purely bearish case separately, which are denoted by $V_{11}(\alpha ; 0.9,1.2)$ and $V_{12}(\alpha ; 0.9,1.2)$, respectively.

Example 4.2. Let $q_{11}=q_{12}=q_{21}=q_{22}=0$ in Example 1 and all the other parameters unchanged. Equations system (3.12) turns out to be (3.13), which can also be solved by the same method as in Example 1. To make a convenient comparison, we plot the regime switching case $V_{1}(\alpha ; 0.9,1.2,1)$ and $V_{2}(\alpha ; 0.9,1.2,2)$ in Figure 5 together with $V_{11}(\alpha ; 0.9,1.2)$ and $V_{12}(\alpha ; 0.9,1.2)$.

We observe in Figure 5 that the purely bullish case the $V$ is the smallest among the four cases. That is because in a bullish market mode, the asset value process is less likely to fall below $\lambda_{1}$, which indicates a less payment from the sponsor. Conversely, in a bearish market mode, more support is needed to guarantee $\lambda_{1}$. However, when the risk of a bad economic situation exists, the sponsor has to increase the support. Then with the 
same starting market mode $i_{0}=1$, the value $V_{1}(\alpha ; 0.9,1.2,1)$ is larger than $V_{11}(\alpha ; 0.9,1.2)$. With $i_{0}=2$, the value $V_{1}(\alpha ; 0.9,1.2,2)$ is even larger. Moreover, due to the similar reason, $V_{12}(\alpha ; 0.9,1.2)$ has the largest value among all the four cases.

\subsection{Optimal dividend}

From the sponsor point of view, an interesting problem is how to optimize the amount paid back to the sponsor. We can think of the payment as dividend, so the problem is related to the optimal dividend problem. De Finetti (1957) studies the optimal dividend problem under a simple discrete model. Subsequently, Gerber (1972). Recently, the topic was discussed extensively by many authors. Some recent papers are Asmussen and Taksar (1997), Højgaard and Taksar (1999), Siegl and Tichy (1999), Højgaard (2002) and Gerber and Shiu $(1998,2004)$. All these authors study the problem in arithmetic growth models for assets and liabilities. Gerber and Shiu (2003) study the problem when the asset value, the liability value, and the dividend barrier are modeled by geometric Brownian motion.

The problem is formulated as follows. Consider a company that pays dividends to its sponsors. The market mode space is still defined by $\mathcal{M}=\{1,2, \ldots, d\}$. The asset value process is modeled by $A(t)$ in $(2.2)$ and the liability value process is modeled by $L_{1}(t)$ in (2.6) (not $L(t)$ here). We adopt a barrier strategy for paying dividends. When $A(t)$ reaches the barrier $L_{2}(t)$ in (2.6), the overflow are paid out as dividends. As a consequence, the original asset values process turns out to be a modified asset value process, which still be denoted by $A^{m}(t)$. The dividends is paid out continuously until the company is "ruined"the time that $A^{m}(t)$ falls below the liability value process $L_{1}(t)$. We are interested in the expectation of the present value of dividends paid out until ruin time, which are denoted by $V\left(\alpha ; \lambda_{1}, \lambda_{2}, i_{0}\right)$ (different meaning from the abbreviated version in Section 3 ) 
for different starting market mode $i_{0} \in \mathcal{M}$.

Since $L_{1}(t), L_{2}(t)$ and $A(t)$ are all geometric Brownian motions, similar to $V_{j}\left(\alpha ; \lambda_{1}, \lambda_{2}, i_{0}\right)$ for $j=1,2$, the function $V\left(\alpha ; \lambda_{1}, \lambda_{2}, i_{0}\right)$ is also homogeneous of degree one with respect to $\alpha, \lambda_{1}$ and $\lambda_{2}$. Based on the same calculation described in Section 3, we have the same system of ordinary differential equations (3.13) for $V\left(\alpha ; \lambda_{1}, \lambda_{2}, i_{0}\right)$. Because $L_{1}(t)$ becomes the ruin barrier and $L_{2}(t)$ is still be the dividend barrier, the boundary condition at $\alpha=\lambda_{1}, \lambda_{2}$ is

$$
\begin{gathered}
V\left(\lambda_{1} ; \lambda_{1}, \lambda_{2}, i_{0}\right)=0, \\
V^{\prime}\left(\lambda_{2} ; \lambda_{1}, \lambda_{2}, i_{0}\right)=1,
\end{gathered}
$$

for $i_{0} \in \mathcal{M}$. Then the solution to (3.13) based on the above boundary conditions is unique. The calculation based on all the parameters is the same as Example 4.1, we plot the solution in Figure 6. We can see that with any initial market mode and with more initial asset value $\alpha$, it is more likely to pay out dividends, that is, $V\left(\alpha ; \lambda_{1}, \lambda_{2}, i_{0}\right)$ is increasing with respect to $\alpha$ under any $i_{0} \in \mathcal{M}$.

In practice, the company has to decide what the $\lambda_{2}$ is based on its time zero liability value $\lambda_{1}$ and asset value $\alpha$. A natural idea is to choose $\lambda_{2}$ in order to maximize the expected present value of all future dividend payments starting from some market mode. That is, with fixed $\alpha, \lambda_{1}$ and $i_{0}$, we choose $\lambda_{2} \in[\alpha,+\infty)$ to maximize $V\left(\alpha ; \lambda_{1}, \lambda_{2}, i_{0}\right)$. In this case, $V\left(\alpha ; \lambda_{1}, \lambda_{2}, i_{0}\right)$ can be seen as a function with respect to the single variable $\lambda_{2}$. However, this optimal $\lambda_{2}$ may not exist. We plot an example in Figure 7 for the parameters given in Example 4.1. We can see that with $\alpha=1$ and $\lambda_{1}=0.9$, the value $V\left(1 ; 0.9, \lambda_{2}, i_{0}\right)$ for $i_{0}=1,2$ keeps increasing with respect to $\lambda_{2}$. That is, we can not 


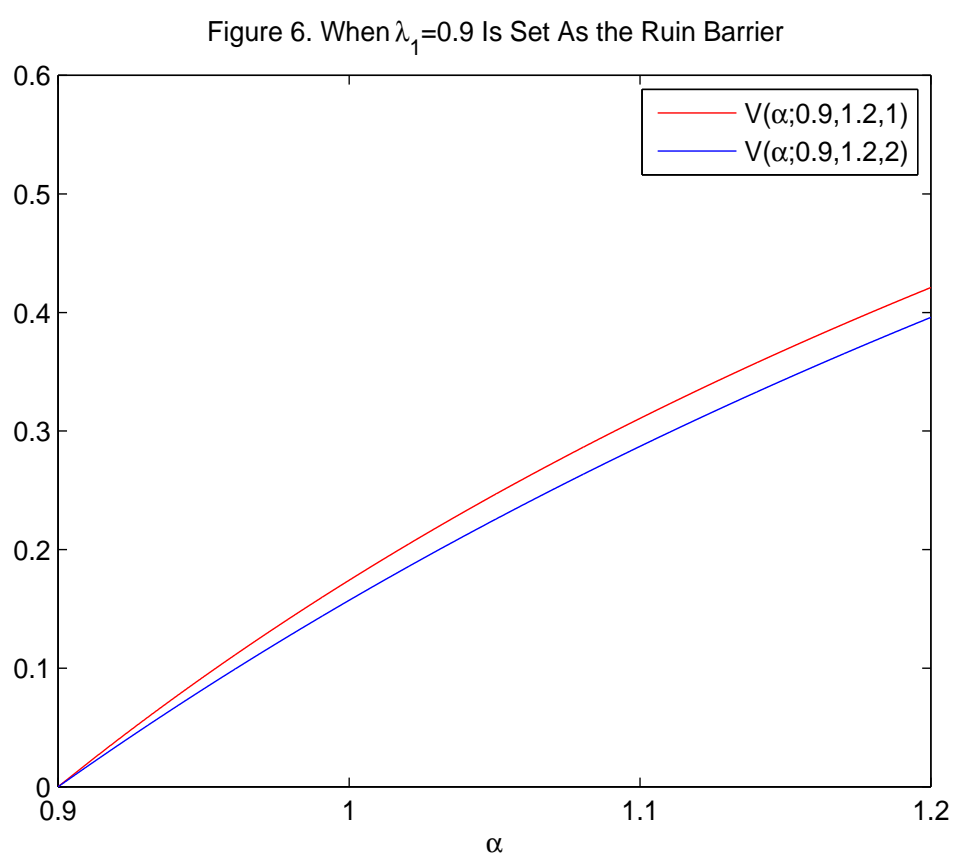

find a finite $\lambda_{2}$ to reach the supreme of the function, which means that there is no need to pay any dividends. This can be explained by observing in Table 1 that the asset growth rate $\mu_{X}$ is higher than the force of interest $\delta$ in both regimes $i_{0}=1,2$, then it is more profitable that all earnings are reinvested in the company's assets. As a result, the increasing profit brings in more chance to pay dividends, which corresponding to an increasing $V\left(\alpha ; \lambda_{1}, \lambda_{2}, i_{0}\right)$ for $i_{0}=1,2$.

By varying some of the parameters in Example 4.1, we find some cases in which the optimal $\lambda_{2}$ is finite. This is illustrated in the next example.

Example 4.3. Let $\lambda_{1}=0.9, \alpha=1, \mu_{X}(1)=0.3, \delta(1)=0.5$ and all the other parameters are the same as that in Example 4.1. We plot $V\left(1 ; 0.9, \lambda_{2}, i_{0}\right)$ for $i_{0}=1,2$ in Figure 8 , which shows clearly that the optimal $\lambda_{2}$ that maximizes the function is finite. 
Figure 7. When $\lambda_{2}$ Is Seen As a Variable

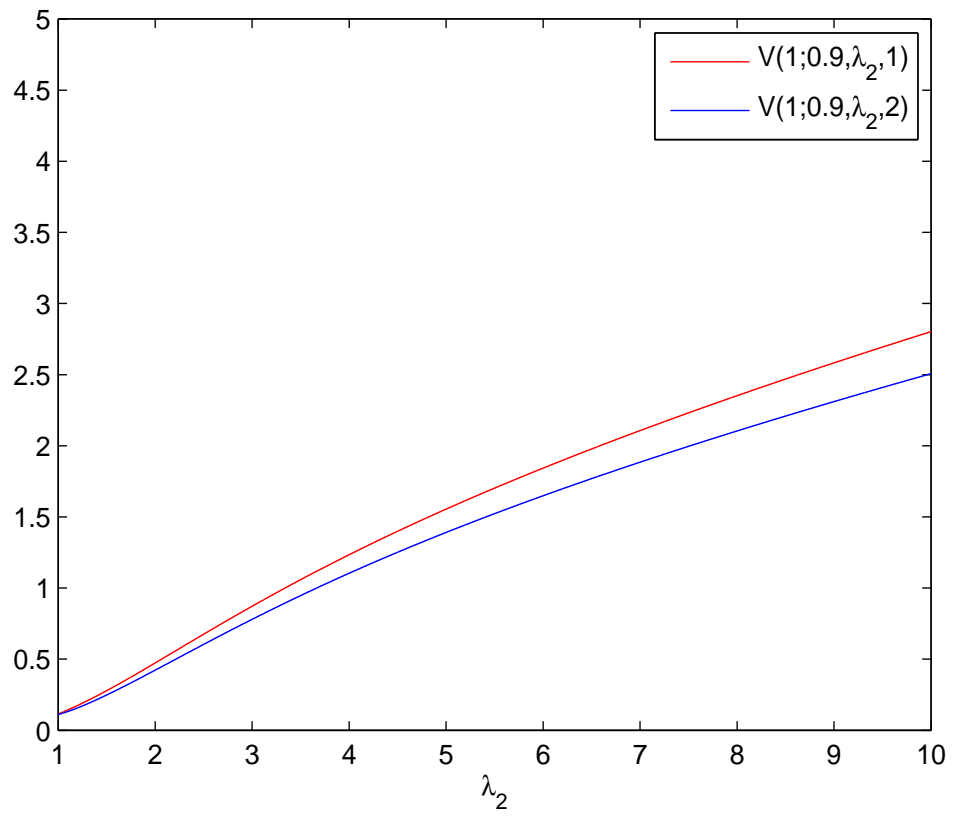

Figure 8. When $\lambda_{2}$ Is Seen As a Variable for Example 4.3

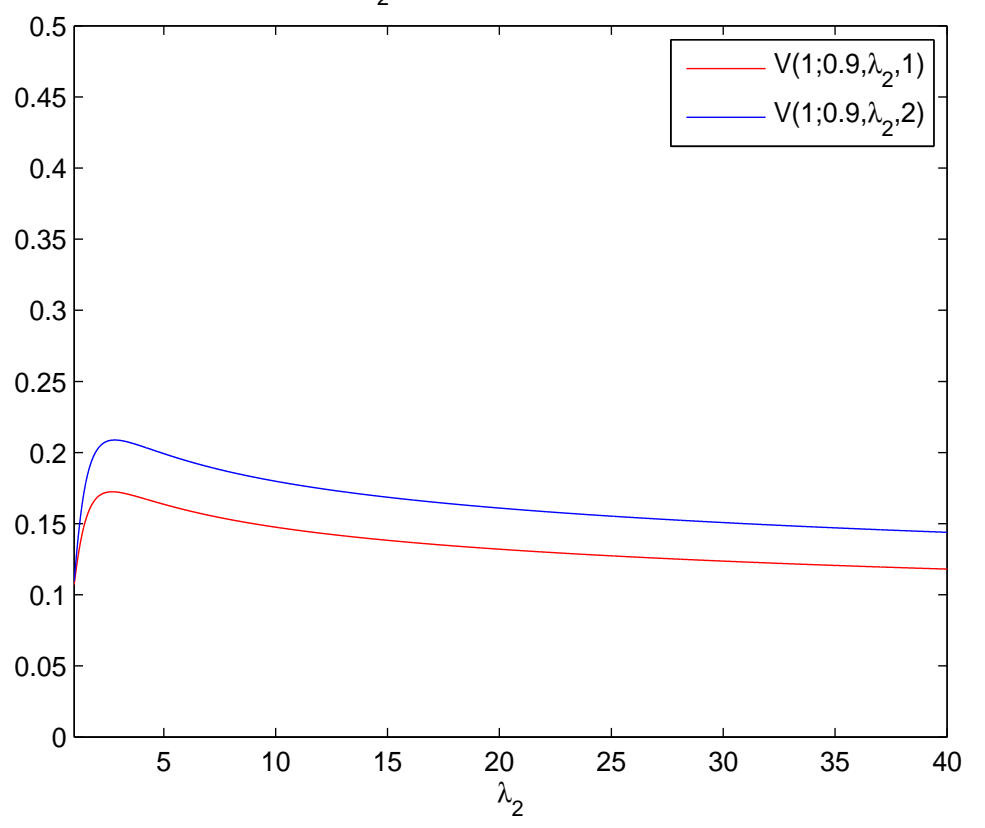




\section{Conclusion}

In this paper, we have extended the model in Gerber and Shiu (2003) to a regime switching version. By adapting the techniques in Gerber and Shiu (2003), systems of differential equations satisfied by $V_{j}\left(\alpha ; \lambda_{1}, \lambda_{2}, i_{0}\right)$ for $j=1,2$ are derived. We have discussed the methodology of how to solve this system of differential equations. We use some numerical examples to illustrate the ideas and methodology.

How to match the asset and liability is one main concern in many asset liability management problems. The problem becomes difficult if the model is more complex and reasonable. There are many interesting problems in this area. We will investigate the problem under a jump-diffusion mode. How to make the model more realistic and some practical aspects of the results are interesting further research topics.

Acknowledgment. The authors would like to thank the referees for valuable comments and suggestions. This research was supported by the Research Grants Council of the Hong Kong Special Administrative Region, China (Project No. 7540/08H)

\section{References}

Asmussen, S., Taksar, M., 1997. Controlled diffusion models for optimal dividend payout. Insurance: Mathematics and Economics 20, 1-15.

Buffington, J., Elliott, R. J., 2002. American options with regime-switching. International Journal of Theoretical and Applied Finance 5, 497-514.

Boyle, P. P., Draviam. T., 2007. Pricing exotic options under regime switching. Insurance: Mathematics and Economics 40 (2), 267-282.

Chen, P., Yang, H., Yin, G., 2008. Markowitz's mean-variance asset-liability management with regime switching: A continuous-time model, Insurance: Mathematics and Economics 43 (3), 456-465. 
De Finetti, B., 1957. Su un' impostazione alternativa dell teoria collettiva del rischio. Transactions of the XVth International Congress of Actuaries 2, 433-443.

Di Masi, G. B., Kabanov, Y. M., Runggaldier, W. J., 1994. Mean variance hedging of options on stocks with Markov volatility. Theory of Probability and its Applications 39 (1), 172-182.

Elliott, R. J., Siu, T. K., 2008a. On Markov-modulated exponential-affine bond price formulae. Applied Mathematical Finance. Accepted

Elliott, R. J., Siu, T. K., 2008b. Robust optimal portfolio choice under Markovian regime-switching model. Methodology and Computing in Applied Probability. Accepted.

Gerber, H. U. 1972. Games of economic survival with discrete and continuous-income process. Operations Research 20 (1), 37-45.

Gerber, H. U., Shiu, E. S. W., 1998. On the time value of ruin. North American Actuarial Journal 2 (1), 48-72. Discussions, 72-78.

Gerber, H. U., Shiu, E. S. W., 2003. Geometric brownian motion models for assets and liabilities: from pension funding to optimal dividends. North American Actuarial Journal 7 (3), 37-51. Discussions, 51-56.

Gerber, H. U., Shiu, E. S. W., 2004. Optimal dividends: analysis with brownian motion. North American Actuarial Journal 8 (1), 1-20.

Hamilton, J. D, 1989. A new approach to the Economic Analysis of Non-stationary Time Series and the Business Cycle, Econometrica 57 (2), 357-384.

Hardy, M. R., 2001. A regime-switching model of long-term stock returns. North American Actuarial Journal 5 (2), 41-53.

Hardy, M. R., 2003. Investment Guarantees: Modeling And Risk Management For Equity-linked Life Insurance. Hoboken, N. J. Wiley.

Højgaard, B., 2002. Optimal dynamic premium control in non-life insurance: maximizing dividend pay-outs. Scandinavian Actuarial Journal 4, 225-245.

Højgaard, B., Taksar, M., 1999. Controlling risk exposure and dividends payout schemes: insurance company example. Mathematical Finance 9, 153-182.

Kim, C., Nelson, C. R., 1999. State-Space Models with Regime Switching: Classical and Gibbs-Sampling Approaches with Applications. Cambridge, Mass: MIT Press.

Lam, P. S, 1990, The Hamilton model with a general autoregressive component, Journal of Monetary Economics 26, 409-432.

Li, S., Lu, Y., 2006. Some optimal dividend problems in a Markov-modulated risk model. University of Melbourne ePrints Repository.

Li, S., Lu, Y., 2007. On the moments of dividend payments and related problems in a Markov-modulated risk model. North American Actuarial Journal 11 (2), 65-76. 
Neftci, S. N. 1984. Are economics time series asymmetric over the business cycle? Journal of Political Economy 92, 307-328.

Quandt, R. E., Henderson, J. M., 1958. Microeconomic Theory: A Mathematical Approach, 2nd edition.

Raymond, J. E., Rich, R. W., 1997. Oil and the macroeconomy: a markov stateswitching approach, Journal of Money, Credit and Banking, 29 (2), 193-213.

Rugemurcia, F. J., 1995. Credibility and changes in policy regime, Journal of Political Economy 103 (1), 176-208.

Siega, T., Tichy, R. E., 1999. A process with stochastic claim frequency and a linear dividend barrier. Insurance: Mathematics and Economics 24, 51-65.

Sotomayor, L., Cadenillas, A., 2008. Classical, singular, and impulse stochastic control for the optimal dividend policy when there is regime switching. Preprint

Storer, P. A., Van Audenrode, M. 1995. Unemployment insurance take-up rates in Canada: facts, determinants and implications. Canadian Journal of Economics 28 (4a), 822-835.

Town, R. J., 1992. Merger waves and the structure of merger and acquisition time series, Journal of Applied Econometrics 7, S83-S100.

Wei, J., Yang, H., Wang, R., 2009. Optimal threshold dividend strategies under the compound Poisson model with regime switching. Submitted.

Zhou, X. Y., Yin, G., 2003. Markowitz's mean-variance portfolio selection with regime switching: A continuous-time model. SIAM Journal on Control and Optimization $42,1466-1482$. 